\title{
Unleashing Potential: Transcranial Direct Current Stimulation over the Right Posterior Parietal Cortex Improves Change Detection in Low-Performing Individuals
}

\author{
Philip Tseng, ${ }^{1 *}$ Tzu-Yu Hsu, ${ }^{1,2 \star}$ Chi-Fu Chang, ${ }^{1}$ Ovid J.L. Tzeng, ${ }^{1,2,3}$ Daisy L. Hung, ${ }^{1,2}$ Neil G. Muggleton, ${ }^{1,4}$ \\ Vincent Walsh, ${ }^{4}$ Wei-Kuang Liang, ${ }^{1}$ Shih-kuen Cheng, ${ }^{1}$ and Chi-Hung Juan ${ }^{1}$ \\ ${ }^{1}$ Institute of Cognitive Neuroscience, National Central University, Jhongli 320, Taiwan, ${ }^{2}$ Institute of Neuroscience, National Yang-Ming University, Taipei \\ 112, Taiwan, ${ }^{3}$ Institute of Linguistics, Academia Sinica, Taipei 115, Taiwan, and ${ }^{4}$ Institute of Cognitive Neuroscience and Department of Psychology, \\ University College London, London WC1N 3AR, United Kingdom
}

The limits of human visual short-term memory (VSTM) have been well documented, and recent neuroscientific studies suggest that VSTM performance is associated with activity in the posterior parietal cortex. Here we show that artificially elevating parietal activity via positively charged electric current through the skull can rapidly and effortlessly improve people's VSTM performance. This artificial improvement, however, comes with an interesting twist: it interacts with people's natural VSTM capability such that low performers who tend to remember less information benefitted from the stimulation, whereas high performers did not. This behavioral dichotomy is explained by event-related potentials around the parietal regions: low performers showed increased waveforms in N2pc and contralateral delay activity (CDA), which implies improvement in attention deployment and memory access in the current paradigm, respectively. Interestingly, these components are found during the presentation of the test array instead of the retention interval, from the parietal sites ipsilateral to the target location, thus suggesting that transcranial direct current stimulation (tDCS) was mainly improving one's ability to suppress no-change distractors located on the irrelevant side of the display during the comparison stage. The high performers, however, did not benefit from tDCS as they showed equally large waveforms in N2pc and CDA, or SPCN (sustained parietal contralateral negativity), before and after the stimulation such that electrical stimulation could not help any further, which also accurately accounts for our behavioral observations. Together, these results suggest that there is indeed a fixed upper limit in VSTM, but the low performers can benefit from neurostimulation to reach that maximum via enhanced comparison processes, and such behavioral improvement can be directly quantified and visualized by the magnitude of its associated electrophysiological waveforms.

\section{Introduction}

Visual short-term memory (VSTM) is a specific memory system that bridges the gap between our detailed but fleeting perceptual memory and high-capacity but time-taxing visual long-term memory. It serves as a mental workspace for complex cognitive tasks (Baddeley, 1986), and consequently the limits to its capacity (Cowan, 2001; Awh et al., 2007) are highly predictive of individual differences in fluid intelligence (Jaeggi et al., 2008; Fukuda et al., 2010). Although VSTM performances vary widely across in-

Received Jan. 25, 2012; revised May 31, 2012; accepted June 7, 2012.

Author contributions: P.T., T.-Y.H., C.-F.C., O.J.L.T., D.L.H., V.W., S.-K.C., and C.-H.J. designed research; P.T. T.-Y.H., C.-F.C., and C.-H.J. performed research; P.T., T.-Y.H., C.-F.C., N.G.M., W.-K.L., S.-K.C., and C.-H.J. contributed unpublished reagents/analytic tools; P.T., T.-Y.H., C.-F.C., N.G.M., W.-K.L., and C.-H.J. analyzed data; P.T., T.-Y.H., C.-F.C., N.G.M., and C.-H.J. wrote the paper.

This work was sponsored by the National Science Council, Taiwan (Grant numbers: 99-2410-H-008-022-MY3, 100-2410-H-008-074-MY3, 99-2911-I-008-025, 100-2511-S-008-009, 98-2410-H-008-010-MY3, 98-2517-S-004001-MY3, and 97-2511-S-008-008-MY5). We are grateful to Ed Vogel and an anonymous reviewer for their constructive comments on the previous versions of this manuscript.

The authors declare no competing financial interests.

*P.T. and T.-Y.H. contributed equally to this work.

Correspondence should be addressed to Dr. Chi-Hung Juan, Institute of Cognitive Neuroscience, National Central University, №. 300, Jhongda Rd., Jhongli City, 320, Taiwan. E-mail: chijuan@cc.ncu.edu.tw.

DOI:10.1523/JNEUROSCI.0362-12.2012

Copyright $\odot 2012$ the authors $\quad 0270-6474 / 12 / 3210554-08 \$ 15.00 / 0$ dividuals (Vogel and Awh, 2008), neuroscientific studies have discovered that activity in the posterior parietal cortex (PPC) is closely associated with one's VSTM performance, and is critically involved throughout all stages of VSTM information processing. For example, during encoding and maintenance stages, fMRI studies have found that activities in the right PPC (rPPC) are positively correlated with VSTM load (Todd and Marois, 2004, 2005; Xu and Chun, 2006), whereas studies using event-related potentials (ERPs) have identified sustained negativity from the parietal cortex that can accurately predict VSTM performance across individuals (Vogel and Machizawa, 2004; Vogel et al., 2005). During memory retrieval and comparison stages, posterior activities also persist when access to VSTM representation is required (Eimer and Kiss, 2010), and patients with PPC damage also exhibit difficulties in VSTM retrieval (Olson and Berryhill, 2008). Thus, this interesting relationship between parietal activity and VSTM performance, as well as how ERP signals from the PPC can predict individual differences, raises the important question of whether VSTM can be artificially and rapidly improved in all individuals by modulating rPPC activity. To this end, in two experiments we applied anodal transcranial direct current stimulation (tDCS) over normal young adults' rPPC to 


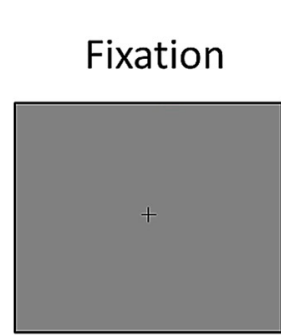

$1000 \mathrm{~ms}$

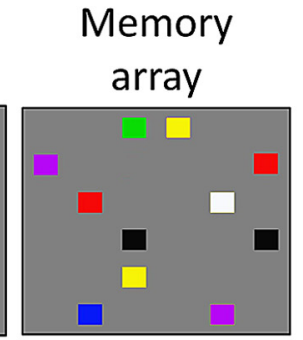

$200 \mathrm{~ms}$
Signal Detection

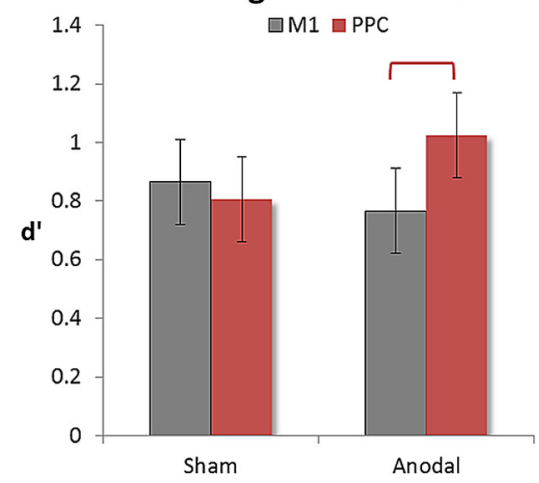

Retention interval

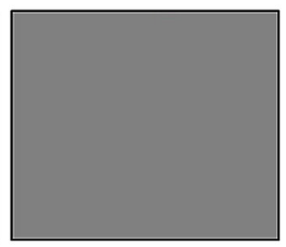

$900 \mathrm{~ms}$

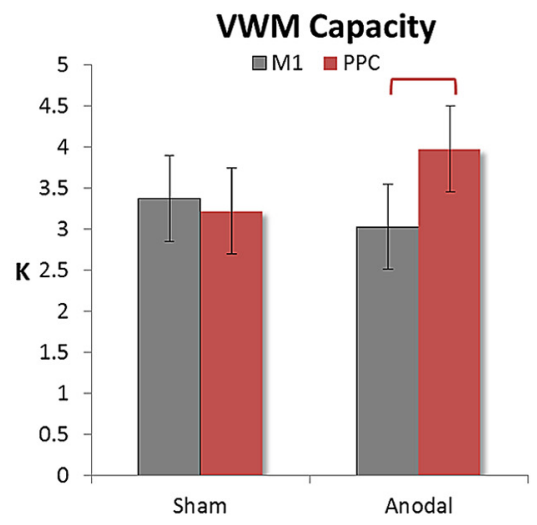

Figure 1. Top, Trial procedure. On half of the trials, one square would change color. Bottom, tDCS-induced improvements in signal detection ( $\left.d^{\prime}\right)$ and Cowan's $K$ in Experiment 1. Change detection performance was evaluated with signal detection analysis and Cowan's $K$. Analyses showed significant increase in $d^{\prime}$ and $K$ in the anodal rPPC TDCS condition. The same tDCS procedure was applied over M1 to rule out any nonspecific effects of tDCS. Error bars represent $95 \%$ confidence intervals.

artificially increase its activity level before performing a VSTM task. The neurophysiology of tDCS, in short, is that anodal (positively charged) stimulation should selectively increase neural spike levels beyond baseline activity by modulating GABAergic activity (Utz et al., 2010), and vice versa for cathodal (negatively charged) stimulation, and the two usually elicits improved or impaired behavioral performance, respectively (Hsu et al., 2011). The technology and application of tDCS is not new (for review, see Nitsche et al., 2008), and similar attempts have been made in the domain of verbal working memory, where tDCS over human prefrontal cortex can induce improvement in memory for digits and words (Fregni et al., 2005; Ohn et al., 2008; Jo et al., 2009; Javadi and Walsh, 2011). However, tDCS over PPC has generated mixed results, such as improved visuospatial orienting (Bolognini et al., 2010a,b) and impaired visual-form memory (Berryhill et al., 2010). Thus, whether VSTM can be artificially improved via tDCS, as well as the underlying mechanisms of such possible effect, are worth investigating. Here we first asked whether humans' VSTM can be improved at all with a short period of electrical current over PPC (Experiment 1) and if so, are there any electrophysiological markers that are predictive of whether someone would receive such memory enhancement (Experiment 2).

\section{Materials and Methods}

To measure the effect of tDCS on VSTM, we used a standard change detection task that required participants to memorize an array of 11 colored rectangles, and compare it with a subsequent display $(900 \mathrm{~ms}$ later) to indicate whether any one rectangle had changed color (Fig. 1, top). This task has been widely used to study VSTM, and provides several quantitative measures that estimate the integrity of memory information at a given moment, making it an ideal candidate to measure quantitative
Test array

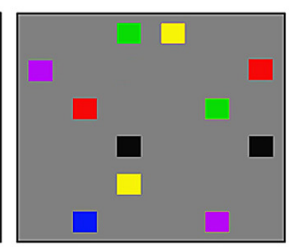

$2200 \mathrm{~ms}$ improvement in VSTM due to anodal electrical stimulation. Importantly, its well defined timeframes for each stage of processing (i.e., encoding, maintenance, retrieval) provides a very strong framework for the application of ERP measurements to investigate underlying cognitive processing.

Participants. Thirty neurologically normal college students with normal or corrected-tonormal vision participated in the experiment, 10 of whom participated in Experiment 1 (5 male, 5 female; mean age $=21$ ) and 20 in Experiment 2 ( 7 male, 13 female; mean age $=22$ ). All gave informed consent before participation. All experimental and tDCS procedures were approved by the Institutional Review Board of the Kaohsiung Medical University Chung-Ho Memorial Hospital, Kaohsiung, Taiwan. The study used a within-subject design, thus each person participated on 2 different days for anodal and sham tDCS sessions. The same design was applied to the primary motor cortex (M1) tDCS study of Experiment 1 , which we describe in more detail below. The order of all sessions was counterbalanced across all participants. We excluded participants whose baseline performance was 2 SDs above or below the mean change detection performance of all participants. Based on this criterion, one participant's data were excluded in Experiment 1.

tDCS protocol. In both experiments we applied tDCS over the right hemisphere because rPPC has been consistently shown to respond to stimuli in both hemifields (Heilman and Van Den Abell, 1980; Beck et al., 2006), and previous TMS studies have found that interfered rPPC, but not 1PPC, activity can lead to impaired change detection (Beck et al., 2006; Tseng et al., 2010). In addition, in our pilot experiments we did not find a reliable effect of cathodal stimulation compared with sham on change detection performance $\left(d^{\prime}: p=0.5 ; K\right.$ : $p=0.55)$, therefore the present study focused on the facilitating effects of anodal stimulation, which typically excites cortical activity, to see whether VSTM performance could be improved with increased rPPC activity.

rPPC was located as P4 according to the international 10-20 system for EEG electrode placement. Since tDCS requires two electrodes that simultaneously stimulate one brain region and inhibit another, the reference electrode was placed on the left cheek (Berryhill et al., 2010; Hsu et al., 2011) to avoid any confounding effect from other brain regions. The current was applied for $15 \mathrm{~min}$ with an intensity of $1.5 \mathrm{~mA}$, which can create an excitatory effect for up to $90 \mathrm{~min}$ (Nitsche and Paulus, 2001). The sham tDCS condition followed an identical procedure, including electrode placements, but stimulation only lasted $30 \mathrm{~s}$. tDCS sessions were performed using a Neuroconn Eldith DC-stimulator and a pair of electrodes housed in $4 \times 4 \mathrm{~cm}$ saline-soaked sponge coverings.

In addition to the sham control condition, we also applied tDCS over M1 in Experiment 1 to control for any tDCS-specific effects. M1 has been investigated in many tDCS studies (Utz et al., 2010) and tDCS has been shown to affect motor-evoked potentials elicited by stimulation of M1. To localize M1, we used TMS to locate the brain region which, when stimulated, would result in a visible finger twitch in the right hand. The tDCS stimulation procedure was identical to the aforementioned PPC condition. As shown in the results (Fig. 1, bottom), the M1 and sham conditions were not statistically different from each other.

ERP protocol. EEG activity was recorded with $\mathrm{Ag} / \mathrm{AgCl}$ electrodes mounted in an elastic cap (Electro-Cap International) using a 64electrode arrangement following the International 10-20 System, offline referenced to the left and right mastoid. Vertical and horizontal electrooculograms were also recorded. Electrode impedances were kept below 5 
$\mathrm{k} \Omega$ for all electrodes, and amplifier bandpass was $0.05-70 \mathrm{~Hz}$. Data were recorded with Neuroscan 4.2 software, with a sampling rate of $1000 \mathrm{~Hz}$. The onset of the test array display was set as the zero point, and epochs ran from -100 to $600 \mathrm{~ms}$. Artifact rejection was performed to exclude trials with eye blinks (EOG amplitude $> \pm 50 \mu \mathrm{V}$ ) or with a movement artifacts (EEG $> \pm 100 \mu \mathrm{V}$ ). Horizontal eye movements were rejected by a two-step procedure as follows: (1) HEOG was rejected when its amplitude was $> \pm 50 \mu \mathrm{V}$, and (2) HEOG was inspected trial-by-trial. According to Luck (2005) (for a brief review, see Woodman, 2010), a bipolar recording of the voltage between electrodes at locations immediately adjacent to the two eyes will yield a deflection of $\sim 16 \mu \mathrm{V}$ for each degree of eye movement. Therefore, in this study a trial was rejected if the peak-to-peak amplitude was larger than $16 \mu \mathrm{V}$.

ERPs from trials containing a change located on either the left or right side were combined by an averaging procedure that preserved the electrode location relative to the target side (ipsilateral or contralateral). $\mathrm{N} 2 \mathrm{pc}$ and SPCN were extracted from the mean differences between contralateral and ipsilateral amplitudes at posterior electrodes PO7/8 (Hopf et al., 2004; Schankin and Wascher, 2007; Eimer and Kiss, 2010). The time windows of N2pc and SPCN were identified from 250 to $320 \mathrm{~ms}$ and from 320 to $600 \mathrm{~ms}$, respectively. The time window for the SPCN component is somewhat later than those reported in other studies using a similar measure of contralateral delay activity (CDA; Vogel and Machizawa, 2004; McCollough et al., 2007), which may be due to the differences between the paradigms, as discussed below.

A two-way repeated-measure ANOVA was computed based on the mean amplitudes of the N2pc and SPCN, testing for the effects of tDCS (anodal and sham) and electrode side (ipsilateral and contralateral to change). This was done for both low and high performers. Only effects including the factor of electrode side, reflecting differential activity observed in contralateral versus ipsilateral electrodes, were of interest.

Experimental design. In both experiments, the behavioral session occurred after tDCS application, regardless of whether the tDCS session was a sham, M1, or rPPC condition. Participants sat $57 \mathrm{~cm}$ in front of the display, rested for 2 min after the stimulation and began the formal behavioral session (with concurrent ERP in Experiment 2). The change detection task consisted of 288 formal trials, half of which contained a change and half did not. Each session lasted $\sim 20 \mathrm{~min}$, which was well within the duration of the expected tDCS effect. Each trial began with a $1000 \mathrm{~ms}$ fixation cross, followed by a $200 \mathrm{~ms}$ memory array, $900 \mathrm{~ms}$ retention interval, and a $2200 \mathrm{~ms}$ test array. It is important to note that, unlike many ERP studies investigating the CDA, we did not embed a directional cue in our paradigm at the onset of the trial. This was done because Experiment 1 was designed to test whether it was possible to improve people's VSTM without ERP recordings. Since we observed an effect from Experiment 1, we conducted Experiment 2 with an identical design to investigate the underlying neurophysiological activities from Experiment 1 . Thus the paradigm here is more similar to a classic change detection paradigm (Luck and Vogel, 1997).

The entire display extended $\sim 31^{\circ} \times 24^{\circ}$, which is bigger than most change detection studies to increase task difficulty and avoid ceiling effects (note that an even bigger version has been used before successfully in a change detection paradigm by Tseng and Bridgeman, 2011). Therefore, the improvement in VSTM performance here may be underestimated due to the bigger display. All trials consisted of 11 rectangles of different colors, sized at $1.6^{\circ}$ wide and $1.3^{\circ} \mathrm{mm}$ tall. All rectangles were kept at least a vertical distance of $1^{\circ}$ and a horizontal distance of $2.4^{\circ}$ apart. Each rectangle was randomly selected from a pool of 7 highly discriminable colors (red, blue, violet, green, yellow, black, and white), and each color could not repeat more than twice within each trial.

Statistical analysis. Signal detection analysis and Cowan's $K$ were used to measure sensitivity to change and assess VSTM performance, respectively. The signal detection equation assumes two normal distributions for signal and noise, and calculates the distance between the two means of the distributions by subtracting their $z$-scores, providing a discrimination index that is independent from one's bias to respond yes or no. The Cowan's $K$ (Cowan, 2001) assumes that an individual can hold $K$ items in memory out of an array of $S$ items such that $K=$ Set Size ${ }^{\star}$ (Hit rate -
False Alarm rate). This equation accounts for guessing by subtracting incorrect guesses from the correct responses.

\section{Results}

\section{Experiment 1: Behavioral results}

The performance of the first group of participants had a rather low variability ( $d^{\prime}$ range: $0.4 \sim 1.25$; $\mathrm{SD}=0.32$ ). Data were analyzed with a repeated-measures $2 \times 2$ ANOVA with factors of tDCS (tDCS vs sham) and stimulation area (PPC vs M1). A significant interaction was observed $\left(F_{(1,8)}=6.39 ; p=0.035\right)$, and separate comparisons revealed that tDCS over rPPC resulted in performance significantly better than sham stimulation $(p=$ $0.038)$ and M1 tDCS ( $p=0.019)$. We also computed Cowan's $K$ and observed the same interaction (Fig. 1, bottom; $F_{(1,8)}=5.93$; $p=0.041)$, where participants held more items in the rPPC tDCS condition than the sham $(p=0.046)$ and M1 tDCS conditions $(p=0.018)$. In addition, we did not observe any difference in hit rates between the left and right hemifield (tDCS: $t_{(8)}=0.92, p=$ 0.38 ; sham: $\left.t_{(8)}=-0.31, p=0.76\right)$.

\section{Experiment 2}

To investigate the electrophysiological mechanisms underlying the tDCS-induced improvement in VSTM seen in Experiment 1, for Experiment 2 we doubled the number of participants and recorded ERPs as they performed the VSTM task, with or without anodal stimulation.

\section{Behavioral results}

In terms of behavioral data, the new group of 20 participants varied widely in their VSTM performance, with $d^{\prime}$ ranging between 0.3 and $1.8(\mathrm{SD}=0.47)$. Comparing these numbers with those from Experiment 1 ( $d^{\prime}$ range: $0.4 \sim 1.25$ ), it is clear that this group included many more high performers (Vogel and Machizawa, 2004) who could hold more items in their VSTM without the help of external stimulation. This apparent difference was also evident in the analyses, as anodal stimulation no longer improved overall performance over the sham condition in terms of $d^{\prime}\left(t_{(19)}=0.3, p=0.77\right)$ and $K\left(t_{(19)}=1.21, p=0.24\right)$ when all participants were grouped together. To examine whether the effect of tDCS interacted with preexisting individual differences in VSTM performance, we sorted the 20 individuals by their $K$ estimates in the sham condition (range: 1.2-5.6 items), and split them by the median score ( $\sim 3.7$ items) (Fukuda and Vogel, 2011). This divided the participants into a high-performing $(n=$ 10 ; mean $\left.d^{\prime}=1.5 ; K=4.6\right)$ and low-performing $(n=10$; mean $d^{\prime}=0.7 ; K=2.3$ ) group based on their natural VSTM capability without $\mathrm{tDCS}$. The ratio between the two groups happened to be 1:2 for both $d^{\prime}$ and $K$. Indeed, when we took the variance of individual differences in VSTM into account, we observed a significant interaction in a $2 \times 2$ ANOVA for tDCS (anodal vs sham) and performance (high vs low) in both $d^{\prime}\left(F_{(1,9)}=8.142, p=\right.$ $0.019)$ and $K\left(F_{(1,9)}=12.37, p=0.007\right)$. Interestingly, subsequent analyses showed that anodal tDCS was only facilitative in the low-performing group (in terms of $K$ at $p=0.002$; $d^{\prime}$ approached significance at $p=0.053$ ), whose performance resembled our participants in Experiment 1, and tDCS was not helpful to the high-performing group $\left(d^{\prime}: p=0.069 ; K: p=0.092 ;\right.$ Fig. 2$)$. In fact, the effect of anodal tDCS on these high performers, if any, seemed to be a detrimental one (Fig. 2) even though statistically this negative trend was only of marginal significance. Thus, more statistical power may be needed to verify how anodal tDCS may have 'squashed' everyone toward the mean, causing low performers to perform significantly better and high performers to per- 

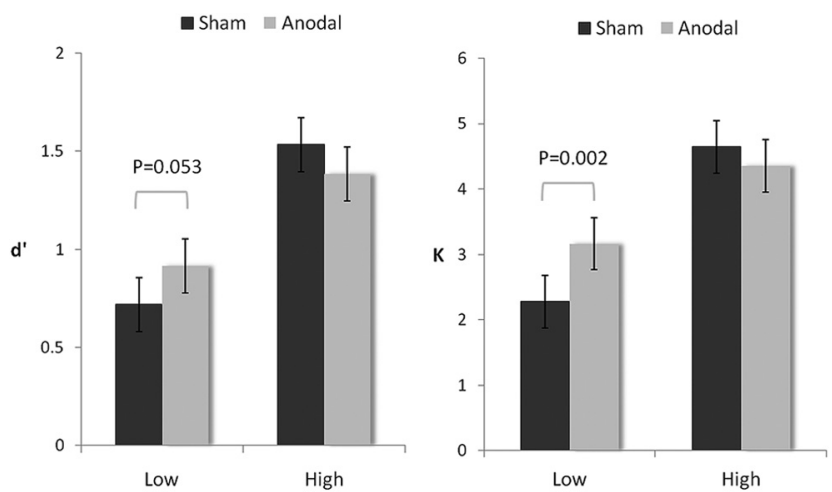

Figure 2. The facilitating effect of anodal tDCS was observed only in low performers. Similar results were obtained for $d^{\prime}$ and $K$. Separate $2 \times 2$ ANOVAs on both measures revealed a main effect of performance $\left[d^{\prime}: F_{(1,9)}=36.22, p<0.01 ; K: F_{(1,9)}=112.11, p<0.01\right]$, no effect of $\operatorname{tDCS}\left(d^{\prime}: F_{(1,9)}=0.088, p=0.773 ; K: F_{(1,9)}=1.45, p=0.25\right)$, and a significant interaction between performance and $\operatorname{tDCS}\left(d^{\prime}: F_{(1,9)}=8.142, p=0.019 ; K: F_{(1,9)}=12.37, p=0.007\right)$. Separate comparisons suggest that the low-performing group received significant improvement from $\operatorname{tDCS}\left(d^{\prime}: p=0.053 ; K: p=0.002\right)$, but high performers did not $\left(d^{\prime}: p=0.069 ; K\right.$ : $p=0.092$ ). Error bars represent $95 \%$ confidence intervals.

form slightly worse. Nonetheless, the current results suggest that anodal tDCS is facilitative only in those individuals whose VSTM performance is below average to begin with. For those high performers who performed well naturally, anodal stimulation did not significantly improve their performance.

$N 2 p c$

ERP results were obtained from both groups to see whether there was an underlying physiological difference between high- and low-performing individuals that could explain their different receptivity to anodal tDCS. We first analyzed N2pc, a negative deflection of the ERP waveform $\sim 200 \mathrm{~ms}$ after stimulus onset (Fig. 3 , top) at posterior sites (PO7 and PO8) contralateral to the attended space. N2pc has been shown to correspond with the deployment of visual attention (Luck and Hillyard, 1994; Eimer, 1996; Woodman and Luck, 1999, 2003), and its amplitude has also been observed to be greater when people detected a change versus when a change is missed (Eimer and Mazza, 2005). Thus, $\mathrm{N} 2 \mathrm{pc}$ has been regarded as an index of the operation of visualspatial attention (Luck and Hillyard, 1994; Eimer, 1996; Woodman and Luck, 2003; Jolicoeur et al., 2008; Woodman et al., 2009; Woodman, 2010). Consistent with previous research (Eimer and Kiss, 2010), we found a greater negative amplitude around PO7 and PO8 when participants detected a change (i.e., hit) than missed one, across both sham $\left(F_{(1,19)}=16.49, p=0.001\right)$ and $\operatorname{tDCS}\left(F_{(1,19)}=7.68, p=0.012\right)$ conditions (Fig. 3, bottom). This $\mathrm{N} 2 \mathrm{pc}$ component was stronger when target appeared in the left visual field (low performers: $p=0.001$; high performers: $p=$ 0.003 ) than the right visual field (low performers: $p=0.068$; high performers: $p=0.256$ ), which is presumably due to the right hemispheric stimulation. Importantly, when we analyzed the effects of tDCS on N2pc in these hit trials, along with participants' natural VSTM capability, we found that only the low-performing group showed an increase in N2pc amplitude from anodal tDCS (Fig. 4, top, first graph). The high performers, on the other hand, already had a large N2pc amplitude to begin with (sham condition), and their N2pc amplitude was not affected by external stimulation (Fig. 4, top, second graph). In summary, the low performers showed a boost to their preexisting N2pc amplitudes as a consequence of anodal tDCS, whereas the high performers
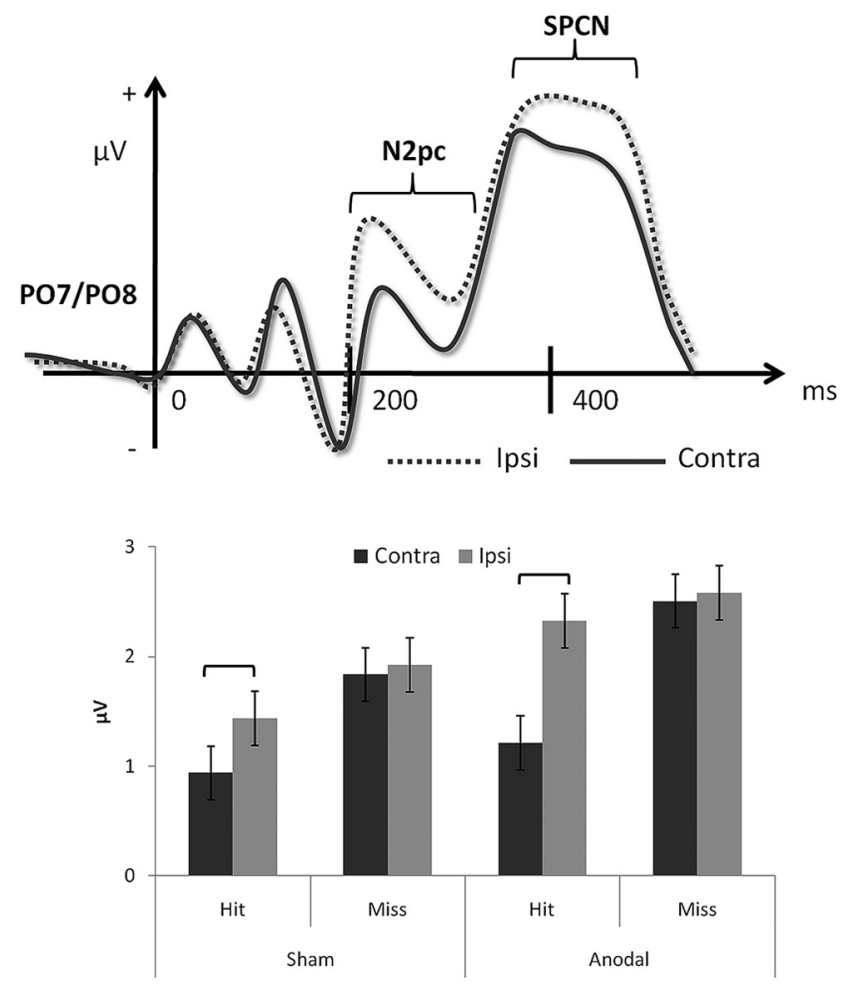

Figure 3. Top, Typical amplitude and timeframe of N2pc and SPCN. Bottom, Only "correct detection" trials showed significant modulation of amplitudes of N2pc. Consistent with previous research, we also found greater N2pc amplitudes when participants detected a change compared with missing one. The ERP data of these hit trials were used to extract N2pc amplitudes and compare the effect of anodal tDCS on low and high performers. Error bars represent $95 \%$ confidence intervals.

who had greater preexisting N2pc amplitudes did not benefit from external stimulation.

\section{SPCN}

In addition to N2pc, we analyzed the SPCN (Jolicoeur et al., 2008), which is also called Contralateral Negative Slow Wave (Klaver et al., 1999), and CDA (Awh et al., 2007; McCollough et al., 2007) by different groups of researchers. SPCN, like N2pc, occurs in the parietal region but begins at a later time point ( $\sim 300 \mathrm{~ms}$ ), following the N2pc (Fig. 3, top). Unlike the N2pc, whose amplitude remains constant regardless of memory load, SPCN amplitude varies according to VSTM load, and remains so throughout the entire retention interval. Thus, SPCN has been proposed to be a reflection of VSTM maintenance (Jolicoeur et al., 2008) and access (Eimer and Kiss, 2010). In the present paradigm, we also observed increased SPCN waveforms due to tDCS, with a weaker left-side advantage (based on target location) than the $\mathrm{N} 2 \mathrm{pc}$ in high performers (left: $p=0.003$; right: $p=$ 0.211 ), and no visual field difference in low performers (left: $p=0.094$; right: $p=0.158$ ). Importantly, in the present paradigm, the SPCN was identified during the presentation of the test array - a time window that is later than the reported timeframe of CDA from most ERP studies (Vogel and Machizawa, 2004; Vogel et al., 2005). In addition to the time of occurrence, the effect of tDCS on SPCN was very much like what was observed for the $\mathrm{N} 2 \mathrm{pc}$. A significant interaction with the effect of tDCS in the low-performing group $\left(F_{(1,9)}=6.635, p=0.03\right)$ was seen because the SPCN emerged only in the tDCS condition but was nonexistent in the sham condition (Fig. 4,bottom, first graph). This interaction was not observed in the high-performing group 
$\left(F_{(1,9)}=0.072, p=0.794\right.$; significant SPCN main effect: $\left.F_{(1,9)}=5.11, p=0.05\right)$ because the amplitude of SPCN was already robust in the sham condition and remained unaltered in the tDCS conditions (Fig. 4, bottom, second graph). Thus, the findings here echo the pattern that was observed from N2pc. However, since the SPCN we have observed here took place in the middle of the comparison stage, following closely behind visual search (i.e., N2pc), it is more likely to be reflecting memory retrieval and access processes (Eimer and Kiss, 2010) in the context of the current paradigm. Together, these ERP results uncover the underlying physiological mechanisms of anodal tDCS by revealing that the increased amplitudes of N2pc and SPCN around $\mathrm{rPPC}$ were direct results of anodal stimulation.

\section{Further ERP analysis}

A closer examination of Figure 4 will reveal that most of the effect in N2pc and SPCN came from a tDCS-induced change in the amplitude of the ipsilateral wave (i.e., the same side as the change, thus in the opposite visual field), while the contralateral amplitude remains mostly unaltered. This is quite surprising, because it suggests that the effect of anodal tDCS is not restricted to enhanced target processing, but mostly due to a stronger electrophysiological activity related to the distractors located on the opposite side of the display. This interesting phenomenon can be understood in terms of the role of distractor suppression in the context of change detection. That is, during the presentation of the test array, the observer must visually search for the target while suppressing other distractors, and simultaneously retrieving and comparing information online. Therefore, successful suppression of the distractors would significantly facilitate the ability to detect the true target and improve change detection performance. This idea of distractor suppression has received ample support from behavioral and ERP studies in the past decade (Vogel and Machizawa, 2004; Vogel et al., 2005; McCollough et al., 2007; Fukuda et al., 2010; Fukuda and Vogel, 2011), and explains the current findings well. Indeed, this effect on the ipsilateral wave was only present in those low performers who showed an improvement $(2 \times 2$ ANOVA: $\operatorname{tDCS}(1,9)=1.528, p=$ 0.248 ; Contralaterality $(1,9)=3.405, p=$ 0.098; Interaction $(1,9)=6.99, p=0.027$; Post hoc: sham contralateral vs anodal contralateral: $p=0.311$; sham ipsilateral
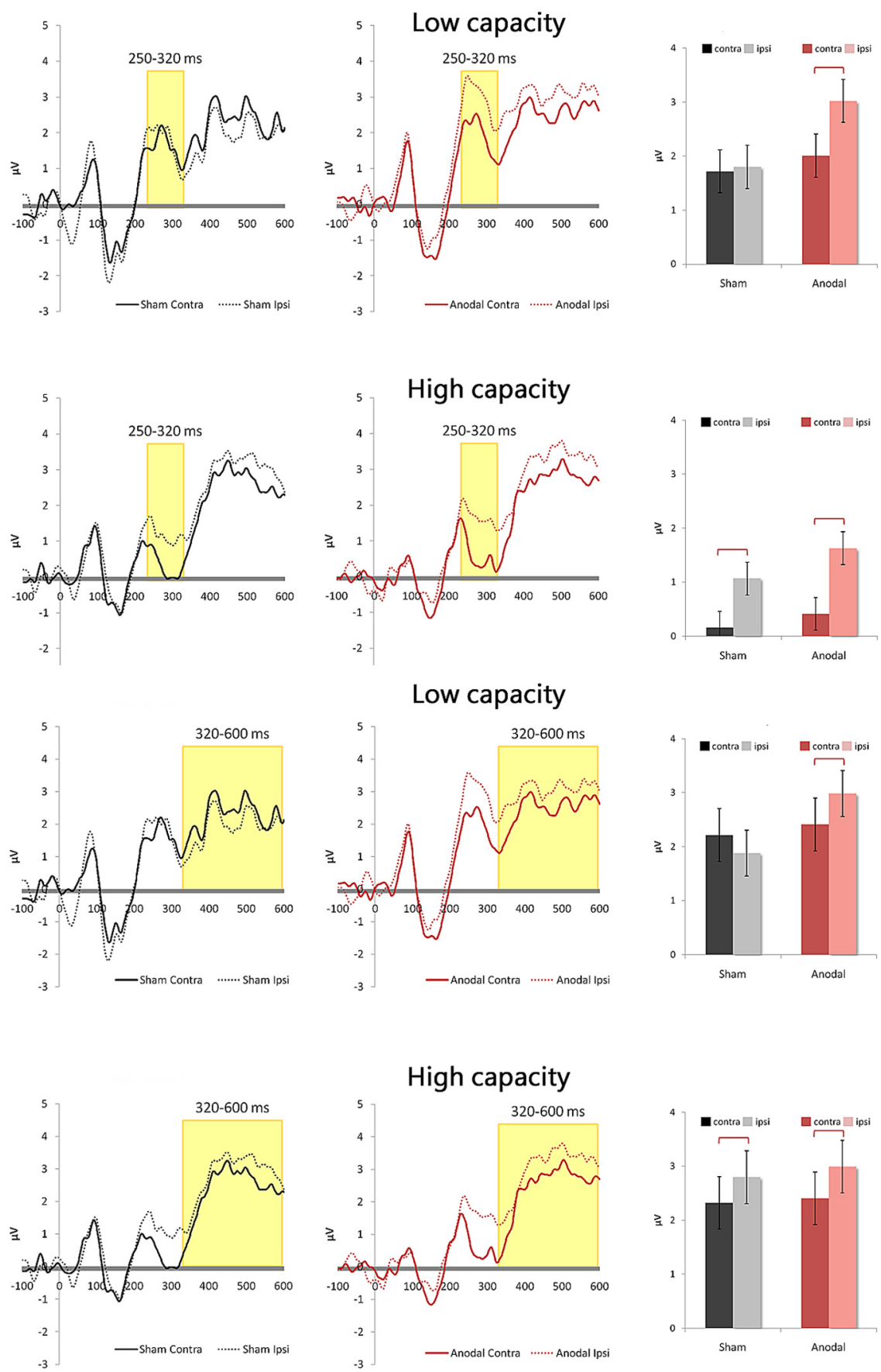

Figure 4. Top, tDCS increases N2pc component in low performers but failed to widen the preexisting gap in high performers The low- and high-performing groups showed a very different reaction to anodal tDCS in their ERP results. In a $2 \times 2$ ANOVA, the low-performing group showed no effect of tDCS $\left(F_{(1,9)}=1.528, p=0.248\right)$, no effect of N2pc $\left(F_{(1,9)}=3.405, p=0.098\right)$, but a significant interaction between $\mathrm{DDCS}$ and $\mathrm{N} 2 \mathrm{pc}\left(F_{(1,9)}=6.99, p=0.027\right)$, which resulted from the N2pc amplitude that emerged in the $\mathrm{tDCS}$ condition ( $p=0.005)$ but not in the sham condition $(p=0.402)$. The high-performing group showed no effect of tDCS $\left(F_{(1,9)}=0.537, p=0.482\right)$ or interaction $\left(F_{(1,9)}=1.29, p=0.285\right)$, but a significant effect of $\mathrm{N} 2 \mathrm{pc}\left(F_{(1,9)}=13.938, p=0.005\right)$, mainly because its N2pc amplitude was apparent with or without anodal tDCS. Error bars represent $95 \%$ confidence intervals. Bottom, Similar patterns in SPCN where low performers benefit from tDCS but high performers do not. Data from SPCN amplitudes reflected what we found from N2pc. The low-performing group showed no effect of $\operatorname{tDCS}\left(F_{(1,9)}=0.579, p=0.46\right)$, no effect of $\operatorname{SPCN}\left(F_{(1,9)}=0.502, p=0.496\right)$, but a significant interaction between $\operatorname{tDCS}$ and $\operatorname{SPCN}\left(F_{(1,9)}=6.635, p=0.03\right)$, which resulted from the SPCN amplitude that appeared in the tDCS condition $(p=0.003)$ but was absent in the sham condition $(p=0.139)$. The high-performing group showed no effect of $\operatorname{tDCS}\left(F_{(1,9)}=0.061, p=0.81\right)$ nor an interaction $\left(F_{(1,9)}=0.072, p=0.794\right)$, but a significant effect of SPCN $\left(F_{(1,9)}=5.11, p=0.05\right)$. Again, this was because SPCN amplitudes were consistent in the sham and tDCS conditions to begin with. Error bars represent $95 \%$ confidence intervals. 
vs anodal ipsilateral: $p=0.056)$ ), lasting all the way from 250 to $600 \mathrm{~ms}$, thus accounting for our earlier behavioral and ERP results and supporting a critical role of distractor suppression during the comparison stage.

\section{Discussion}

The behavioral and ERP results here suggest that the well documented upper limit to change detection performance cannot be easily broken, at least for high-performing individuals who are already above average to begin with. As such, these high performers did not, or could not, push the associated electrophysiological components, the N2pc and SPCN, to higher amplitudes with our current stimulation parameters. Intriguingly, for the low performers who did not originally show elevated N2pc and SPCN amplitudes, a simple session of 15 min anodal tDCS over rPPC was able to improve their VSTM and change detection performance. Thus, although anodal tDCS in theory should result in facilitation compared with cathodal stimulation, may be more or less dependent on people's natural ability and an upper limit on performance-something future tDCS studies should carefully consider.

\section{Interaction between tDCS and natural ability}

Past research has demonstrated that the wide range of individual differences in VSTM can be predicted from each person's level of parietal activation (Todd and Marois, 2004, 2005; Awh et al., 2007; McCollough et al., 2007). Our results here suggest that the low performers do not actually suffer from a low VSTM capacity per se (Vogel et al., 2005), but rather, their poor change detection performance may be a direct result of failure to show high amplitudes of N2pc and SPCN that are associated with facilitated cognitive and neural processes, especially during the retrieval and comparison stages in the present study. To this end the anodal tDCS was able to artificially increase their rPPC activation and elevate their N2pc and SPCN amplitudes to similar levels as the high performers and improve performance. Although, it is worthy of note that despite similar amplitudes in N2pc and SPCN after tDCS, the low performers' new and improved performance was still not quite on par with their high-performing counterparts (Fig. 2, bottom). This may be due to the "real" idiosyncratic limitations in change detection, or simply insufficient stimulation from tDCS, both of which await further investigation. Likewise, whether or not the high-performing individuals can improve beyond their above-average performance also remains an open topic for future studies. Future work should also disambiguate the effects of tDCS on different memory systems, as well as the kind of information that is subject to enhanced retrieval and comparison.

\section{An attentional account of the effects of tDCS}

It is important to note that the current study observed a significant tDCS-induced improvement in memory comparisons of color-location bound representations (Luck and Vogel, 1997; Xu and Chun, 2006; Awh et al., 2007; Bays et al., 2011; Brady et al., 2011), while other studies have used nonspatial and uniformly colored stimuli with mixed results (Nitsche et al., 2008; Berryhill et al., 2010; Utz et al., 2010). This may be due to rPPC's sensitivity to spatially configured stimuli, or that memory of objectdrawings is more prone to participants' previous experiences than simple gist-free colored squares. Importantly, the amplitude of SPCN has been shown to be sensitive to stimulus set size within a short timeframe (Jolicoeur et al., 2008), which may explain why studies using longer exposure times may not capture the window of increased SPCN amplitude. Nevertheless, the results here suggest that the ability to elevate visual attention (N2pc) and memory access (SPCN) processes in $\mathrm{PPC}$ may be an important step to explaining individual differences in VSTM performance. Indeed, the tDCS-induced improvement here is most likely attentional rather than VSTM-specific (Desimone and Duncan, 1995; Desimone, 1996; Awh et al., 2000, 2006; Awh and Jonides, 2001; Woodman et al., 2007; Cusack et al., 2009; Berryhill et al., 2011; Linke et al., 2011; Gladwin et al., 2012), as the functional roles of PPC have been well established in updating spatial mapping (Morris et al., 2007; Prime et al., 2008), directing attention to salient stimuli (Nobre, 2001; Rushworth and Taylor, 2006; Chao et al., 2011), binding features (Friedman-Hill et al., 1995; Ellison et al., 2007), monitoring temporal patterns (Coull and Nobre, 1998), deploying top-down attentional bias (Kastner and Ungerleider, 2001; Pessoa and Ungerleider, 2004), and VSTM maintenance (Todd and Marois, 2004, 2005; Vogel and Machizawa, 2004; Tseng et al., 2010) and retrieval (Olson and Berryhill, 2009; Eimer and Kiss, 2010; Berryhill, 2012). Thus, an attentional boost, whether externally via tDCS or internally by other means (Tseng and Bridgeman, 2011) will indeed predict an improvement in VSTM performance, and is very compatible with previous ERP findings on VSTM (Vogel and Machizawa, 2004; McCollough et al., 2007) and change detection (Pourtois et al., 2006). Indeed, Vogel et al. (2005) have demonstrated that although SPCN during the retention interval varies with memory load, efficient filtering of irrelevant information can effectively prevent SPCN from prematurely reaching its asymptotic level by reducing the amount of attentional and memory resources that are used for unnecessary processing. Thus, the real underlying factor that differentiates the high and low performers may lie in their ability to attentionally prioritize relevant information and suppress the distractors. The present findings strongly support this view. As noted earlier, a large portion of the tDCS effect was driven by the ipsilateral (relative to the target) activity, which becomes much more positive with tDCS. Since the hemisphere that is ipsilateral to the target is involved in processing of the visual field located on the opposite side to the target, this suggests that the effect of tDCS is eliciting a stronger suppression of the opposite visual field and distractors. This filtering account makes sense especially in the context of the test array, the time window where we observed the effect of tDCS on N2pc and SPCN, which has also been shown to relate to comparison processes that can contribute as a potential source of apparent capacity limits in VSTM (Awh et al., 2007; Hyun et al., 2009; Makovski et al., 2010). That is, during the test array, one must actively search for the target $(\mathrm{N} 2 \mathrm{pc})$ while accessing memory representations (SPCN) for valid comparisons. Furthermore, previously compared stimuli (i.e., distractors) must be suppressed, or disengaged from, to facilitate efficient search toward the next item until the target is found. Indeed, Sauseng et al. (2009) demonstrated that repetitive TMS at $10 \mathrm{~Hz}$ over PPC ipsilateral to the target can produce increased suppression of irrelevant items on the contralateral side, with a detrimental effect to VSTM when applied contralaterally. Thus, the increased ipsilateral activity from the present results supports the idea that filtering and suppression of irrelevant information may also be an important factor in explaining individual differences in VSTM. On a related note, although the SPCN component we have observed here during the comparison stage looks strikingly similar to the CDA component that many have observed during the maintenance period from the same parietal sites, it remains an open question as to whether they are the same component or not. At the very least, they seem to be 
functionally different, because the memory maintenance demand during the retention interval is quite different from the retrieval and comparison processes during the test array. However, from an attentional perspective, these two components may be functionally different yet similar in terms of their attentional demand. It also remains an open question whether CDA during the retention interval can be altered by tDCS. Here we could not observe any effect during the retentional interval due to the paradigm difference, thus the present study can only conclude that tDCS enhanced one's VSTM retrieval or comparison processes to improve change detection performance.

Many studies have documented a wide range of VSTM performance across different individuals. Here we report that although the upper limit to VSTM seems to be fixed, people who perform below average are able to improve when rPPC activity is increased. This effect requires no training, is immediate and effortless, but most importantly, it can be traced to its direct impact on the electrophysiological processes underlying visual attention and VSTM retrieval and comparison. These results, for the first time, reveal a close relationship between people's natural ability and their receptivity to artificial electrical stimulation. They also demonstrate a critical role for $\mathrm{N} 2 \mathrm{pc}$ and SPCN during the comparison stage as an index of improvements to VSTM performance.

\section{References}

Awh E, Jonides J (2001) Overlapping mechanisms of attention and spatial working memory. Trends Cogn Sci 5:119-126.

Awh E, Anllo-Vento L, Hillyard SA (2000) The role of spatial selective attention in working memory for locations: evidence from event-related potentials J Cogn Neurosci 12:840-847.

Awh E, Vogel EK, Oh SH (2006) Interactions between attention and working memory. Neuroscience 139:201-208.

Awh E, Barton B, Vogel EK (2007) Visual working memory represents a fixed number of items regardless of complexity. Psychol Sci 18:622-628.

Baddeley AD (1986) Working memory. Oxford UK: Clarendon.

Bays PM, Wu EY, Husain M (2011) Storage and binding of object features in visual working memory. Neuropsychologia 49:1622-1631.

Beck DM, Muggleton N, Walsh V, Lavie N (2006) Right parietal cortex plays a critical role in change blindness. Cereb Cortex 16:712-717.

Berryhill ME (2012) Insights from neuropsychology: pinpointing the role of the posterior parietal cortex in episodic and working memory. Front Integr Neurosci 6:1-12.

Berryhill ME, Wencil EB, Branch Coslett H, Olson IR (2010) A selective working memory impairment after transcranial direct current stimulation to the right parietal lobe. Neurosci Lett 479:312-316.

Berryhill ME, Chein J, Olson IR (2011) At the intersection of attention and memory: the mechanistic role of the posterior parietal lobe in working memory. Neuropsychologia 49:1306-1315.

Bolognini N, Fregni F, Casati C, Olgiati E, Vallar G (2010a) Brain polarization of parietal cortex augments training-induced improvement of visual exploratory and attentional skills. Brain Res 1349:76-89.

Bolognini N, Olgiati E, Rossetti A, Maravita A (2010b) Enhancing crossmodal audiovisual processing by brain polarization of the parietal cortex. Eur J Neurosci 31:1800-1806.

Brady TF, Konkle T, Alvarez GA (2011) A review of visual memory capacity: beyond individual items and toward structured representations. J Vis 11:1-34.

Chao CM, Tseng P, Hsu TY, Su JH, Tzeng OJ, Hung DL, Muggleton NG, Juan CH (2011) Predictability of saccadic behaviors is modified by transcranial magnetic stimulation over human posterior parietal cortex. Hum Brain Mapp 32:1961-1972.

Coull JT, Nobre AC (1998) Where and when to pay attention: the neural systems for directing attention to spatial locations and to time intervals as revealed by both PET and fMRI. J Neurosci 18:7426-7435.

Cowan N (2001) The magical number 4 in short-term memory: a reconsideration of mental storage capacity. Behav Brain Sci 24:87-185.

Cusack R, Lehmann M, Veldsman M, Mitchell DJ (2009) Encoding strategy and not visual working memory capacity correlates with intelligence. Psychon Bull Rev 16:641-647.

Desimone R (1996) Neural mechanisms for visual memory and their role in attention. Proc Natl Acad Sci U S A 93:13494-13499.

Desimone R, Duncan J (1995) Neural mechanisms of selective visual attention. Annu Rev Neurosci 18:193-222.

Eimer M (1996) The N2pc component as an indicator of attentional selectivity. Electroencephalogr Clin Neurophysiol 99:225-234.

Eimer M, Kiss M (2010) An electrophysiological measure of access to representations in visual working memory. Psychophysiology 47:197-200.

Eimer M, Mazza V (2005) Electrophysiological correlates of change detection. Psychophysiology 42:328-342.

Ellison A, Lane AR, Schenk T (2007) The interaction of brain regions during visual search processing as revealed by transcranial magnetic stimulation. Cereb Cortex 17:2579-2584.

Fregni F, Boggio PS, Nitsche M, Bermpohl F, Antal A, Feredoes E, Marcolin MA, Rigonatti SP, Silva MT, Paulus W, Pascual-Leone A (2005) Anodal transcranial direct current stimulation of prefrontal cortex enhances working memory. Exp Brain Res 166:23-30.

Friedman-Hill SR, Robertson LC, Treisman A (1995) Parietal contributions to visual feature binding: evidence from a patient with bilateral lesions. Science 269:853-855.

Fukuda K, Vogel EK (2011) Individual differences in recovery time from attentional capture. Psychol Sci 22:361-368.

Fukuda K, Vogel E, Mayr U, Awh E (2010) Quantity not quality: the relationship between fluid intelligence and working memory capacity. Psychon Bull Rev 17:673-679.

Gladwin TE, den Uyl TE, Fregni FF, Wiers RW (2012) Enhancement of selective attention by tDCS: interaction with interference in a Sternberg task. Neurosci Lett 512:33-37.

Heilman KM, Van Den Abell T (1980) Right hemisphere dominance for attention. Neurology 30:327-330.

Hopf JM, Boelmans K, Schoenfeld MA, Luck SJ, Heinze HJ (2004) Attention to features precedes attention to locations in visual search: evidence from electromagnetic brain responses in humans. J Neurosci 24:1822-1832.

Hsu TY, Tseng LY, Yu JX, Kuo WJ, Hung DL, Tzeng OJ, Walsh V, Muggleton NG, Juan CH (2011) Modulating inhibitory control with direct current stimulation of the superior medial frontal cortex. Neuroimage 56:2249-2257.

Hyun JS, Woodman GF, Vogel EK, Hollingworth A, Luck SJ (2009) The comparison of visual working memory representations with perceptual inputs. J Exp Psychol Hum Percept Perform 35:1140-1160.

Jaeggi SM, Buschkuehl M, Jonides J, Perrig WJ (2008) Improving fluid intelligence with training on working memory. Proc Natl Acad Sci U S A 105:6829-6833.

Javadi AH, Walsh V (2011) Transcranial direct current stimulation (tDCS) of the left dorsolateral prefrontal cortex modulates declarative memory. Brain Stimul. Advance online publication. Retrieved July 26, 2011. doi:10.1016/j.brs.2011.06.007.

Jo JM, Kim YH, Ko MH, Ohn SH, Joen B, Lee KH (2009) Enhancing the working memory of stroke patients using tDCS. Am J Phys Med Rehabil 88:404-409.

Jolicoeur P, Brisson B, Robitaille N (2008) Dissociation of the N2pc and sustained posterior contralateral negativity in a choice response task. Brain Res 1215:160-172.

Kastner S, Ungerleider LG (2001) The neural basis of biased competition in human visual cortex. Neuropsychologia 39:1263-1276.

Klaver P, Talsma D, Wijers AA, Heinze HJ, Mulder G (1999) An eventrelated brain potential correlate of visual short-term memory. Neuroreport 10:2001-2005.

Linke AC, Vicente-Grabovetsky A, Mitchell DJ, Cusack R (2011) Encoding strategy accounts for individual differences in change detection measures of VSTM. Neuropsychologia 49:1476-1486.

Luck SJ (2005) An introduction to the event-related potential technique. Cambridge, MA: MIT.

Luck SJ, Hillyard SA (1994) Effects of spatial cuing on luminance detectability: psychophysical and electrophysiological evidence for early selection. J Exp Psychol Hum Percept Perform 20:1000-1014.

Luck SJ, Vogel EK (1997) The capacity of visual working memory for features and conjunctions. Nature 390:279-281.

Makovski T, Watson LM, Koutstaal W, Jiang YV (2010) Method matters: 
systematic effects of testing procedure on visual working memory sensitivity. J Exp Psychol Learn Mem Cogn 36:1466-1479.

McCollough AW, Machizawa MG, Vogel EK (2007) Electrophysiological measures of maintaining representations in visual working memory. Cortex 43:77-94.

Morris AP, Chambers CD, Mattingley JB (2007) Parietal stimulation destabilizes spatial updating across saccadic eye movements. Proc Natl Acad Sci U S A 104:9069-9074.

Nitsche MA, Cohen LG, Wassermann EM, Priori A, Lang N, Antal A, Paulus W, Hummel F, Boggio PS, Fregni F, Pascual-Leone A. (2008) Transcranial direct current stimulation: State of the art 2008. Brain Stimul 1:206-223.

Nitsche MA, Paulus W (2001) Sustained excitability elevations induced by transcranial DC motor cortex stimulation in humans. Neurology 57:1899-1901.

Nobre AC (2001) The attentive homunculus: now you see it now you don't. Neurosci Biobehav Rev 25:477-496.

Ohn SH, Park CI, Yoo WK, Ko MH, Choi KP, Kim GM, Lee YT, Kim YH (2008) Time-dependent effect of transcranial direct current stimulation on the enhancement of working memory. Neuroreport 19:43-47.

Olson IR, Berryhill M (2009) Some surprising findings on the involvement of the parietal lobe in human memory. Neurobiol Learn Mem 91:155-165.

Pessoa L, Ungerleider LG (2004) Neural correlates of change detection and change blindness in a working memory task. Cereb Cortex 14:511-520.

Pourtois G, De Pretto M, Hauert CA, Vuilleumier P (2006) Time course of brain activity during change blindness and change awareness: performance is predicted by neural events before change onset. J Cogn Neurosci 18:2108-2129.

Prime SL, Vesia M, Crawford JD (2008) Transcranial magnetic stimulation over posterior parietal cortex disrupts transsaccadic memory of multiple objects. J Neurosci 28:6938-6949.

Rushworth MF, Taylor PC (2006) TMS in the parietal cortex: updating representations for attention and action. Neuropsychologia 44:2700-2716.

Sauseng P, Klimesch W, Heise KF, Gruber WR, Holz E, Karim AA, Glennon M, Gerloff C, Birbaumer N, Hummel FC (2009) Brain oscillatory substrates of visual short-term memory capacity. Curr Biol 19:1846-1852.

Schankin A, Wascher E (2007) Electrophysiological correlates of stimulus processing in change blindness. Exp Brain Res 183:95-105.
Todd JJ, Marois R (2004) Capacity limit of visual short-term memory in human posterior parietal cortex. Nature 428:751-754.

Todd JJ, Marois R (2005) Posterior parietal cortex activity predicts individual differences in visual short-term memory capacity. Cogn Affect Behav Neurosci 5:144-155.

Tseng P, Bridgeman B (2011) Improved change detection with nearby hands. Exp Brain Res 209:257-269.

Tseng P, Hsu TY, Muggleton NG, Tzeng OJ, Hung DL, Juan CH (2010) Posterior parietal cortex mediates encoding and maintenance processes in change blindness. Neuropsychologia 48:1063-1070.

Utz KS, Dimova V, Oppenländer K, Kerkhoff G (2010) Electrified minds: transcranial direct current stimulation (tDCS) and Galvanic Vestibular Stimulation (GVS) as methods of non-invasive brain stimulation in neuropsychology - a review of current data and future implications. Neuropsychologia 48:2789-2810.

Vogel E, Awh E (2008) How to exploit diversity for scientific gain: using individual differences to constrain cognitive theory. Curr Dir Psychol Sci 17:171-176.

Vogel EK, Machizawa MG (2004) Neural activity predicts individual differences in visual working memory capacity. Nature 428:748-751.

Vogel EK, McCollough AW, Machizawa MG (2005) Neural measures reveal individual differences in controlling access to working memory. Nature 438:500-503.

Woodman GF (2010) A brief introduction to the use of event-related potentials in studies of perception and attention. Atten Percept Psychophys 72:2031-2046.

Woodman GF, Luck SJ (1999) Electrophysiological measurement of rapid shifts of attention during visual search. Nature 400:867-869.

Woodman GF, Luck SJ (2003) Serial deployment of attention during visual search. J Exp Psychol Hum Percept Perform 29:121-138.

Woodman GF, Luck SJ, Schall JD (2007) The role of working memory representations in the control of attention. Cereb Cortex 17 [Suppl 1]: $118-124$.

Woodman GF, Arita JT, Luck SJ (2009) A cuing study of the N2pc component: an index of attentional deployment to objects rather than spatial locations. Brain Res 1297:101-111.

Xu Y, Chun MM (2006) Dissociable neural mechanisms supporting visual short-term memory for objects. Nature 440:91-95. 